\section{Data de la producción científica académica 1940 - 2014 e insumo para la calidad universitaria. Facultad de Odontología de la Universidad Nacional Mayor de San Marcos}

\author{
Data of academic scientific production from 1940 to 2014 and input for univer- \\ sity quality. Dental faculty of Universidad Nacional Mayor de San Marcos
}

\section{Resumen}

Con el objetivo de aportar al mayor conocimiento de la producción científica académica de la Facultad de Odontología de la Universidad Nacional Mayor de San Marcos, se muestra la producción numérica longitudinal retrospectiva entre 1940 y 2014, de las 2833 Tesis de Bachiller, 74 de Maestría, 41 de Doctorado y 154 de Segunda Especialización. La información también es un insumo factual final para los indicadores de la Calidad universitaria. Se sugieren potencialidades de aprovechamiento de tal volumen de fuentes de conocimientos científicos.

Palabras clave: Tesis bachiller, maestría, doctoral, segunda especialización, indicador de calidad.

\section{Abstract}

In order to contribute to better understanding of the academic scientific production of the Faculty of Dentistry at Universidad Nacional Mayor de San Marcos, we present the numerical longitudinal retrospective production between 1940 - 2014, there are 2,833 Bachelor thesis, 74 MSc, 41 Doctoral and 154 Second Specialization. This information is also a final factual input for indicators of university quality. Potential uses of such a volume of sources of scientific knowledge are suggested.

Keywords: Bachelor thesis; mastery; doctoral; second specialization; quality indicator.

\section{Artículo ORIGINAL}

Hilda Moromi-Nakata1, Patricia Delgado Mejía ${ }^{2}$

1. Departamento Académico de Ciencias Básicas de la Facultad de Odontología de la Universidad Nacional Mayor de San Marcos, Perú.

2. Biblioteca de la Facultad de Odontología de la Universidad Nacional Mayor de San Marcos, Perú.

Correspondencia:

Biólogo, Mg Ad Educ. Hilda Moromi-Nakata. Facultad de Odontología de la Universidad Nacional Mayor de San Marcos. Av Germán Amézaga 375, Lima 01. Perú.

Correo electrónico: hmnbio@hotmail.com

\section{Introducción}

La producción científica académica de las instituciones universitarias se encuentran plasmadas en las Tesis de los Grados (Bachiller, Maestro y Doctor), así como en las de Segunda Especialización. Están ubicadas en las bibliotecas, para los usos pertinentes, pero también como un archivo institucional y una evidencia de la cronología de la producción científica académica.

Tal masa de Tesis catalogadas y ubicadas en los anaqueles bibliotecarios es una importante fuente de investigación, lamentablemente desaprovechadas en las universidades. Hay una multitud de variables para optar y plantear diversas investigaciones, para saberes del pasado y orientar el futuro.

El presente es un estudio descriptivo retrospectivo de la data o registro numé- rico de las Tesis de Bachiller, Maestro, Doctor y de Segunda Especialización, en el lapso de 1940 - 2014.

En el mundo de la Tesis universitarias, esta es apenas una de las investigaciones más elementales, conformante de la punta del "iceberg", en cuyo fondo ignoto quedan el análisis y evaluación de los valiosos saberes de las miles de Tesis.

\section{Evidencias}

El conjunto de las evidencias numéricas ahora también toman sentido de actualidad, porque constituyen uno de los insumos cuantitativos de los indicadores para la acreditación universitaria. ${ }^{1}$ Por ejemplo, se podrá confrontar las cantidades de Tesis, en el lugar de uso social (la biblioteca), en cada una de los gestiones institucionales (Decanos, Directores).
Las cuantías han sido procesadas a partir de los archivos de la hemeroteca de la Facultad. ${ }^{2}$ En el gráfico 1, se muestra la cantidad 2833 de Tesis de Bachiller, producidas en el lapso de 1940 al 2014 (la primera, pertenece a Clemente Del Águila, con el tema: Higiene bucal), organizados en quinquenios, para facilitar el análisis numérico y tendencias históricas. Por ejemplo se aprecia:

1. Que el 75,39\% se ha producido entre 1940 - 1975, mientras que el $24,61 \%$ restante en el período 1976 - 2014. ¿’Por qué?:

2. En el primer lapso: 1) por los muchos graduandos, en una de las pocas Facultades del país, y 2) por la obligatoriedad de hacer una tesis para la profesionalización.

3. Luego de la segunda mitad de los sesentas se aprecia una tendencia 
decreciente, que seguro refleja la menor cuantía estudiantil, debido a la proliferación de Facultades en el sistema universitario peruano.

4. Se aprecia un hipo (que puede ser "cero") en el quinquenio 19911995, que debe ser el reflejo de "Bachillerato automático". Sin embargo, después del cual, se ha seguido produciendo tesis; lo que seguramente refleja la "cultura" de la institución sanmarquina: hacer una Tesis a pesar de la implemen- tación de cursos de titulación, que actualmente ya se encuentran eliminados en la Facultad.

En el gráfico 1, se ha insertado la información de las 41 Tesis Doctorales, producto de investigaciones personales, sin antecedente de formación escolarizada. Se nota que aparecen en la segunda mitad de los 40, y alcanzan su pico en el segundo quinquenio de los 70 .

En el gráfico 2, se muestra el historial numérico de las 74 Tesis de Maestría.
Se aprecia que se inician en 1992, con un pico en 1998 y con ausencias en el 2006 y 2009.

Se muestra también la data histórica de las 154 "Tesis" de la Segunda Especialización, que se inicia en 1997, y después una tendencia creciente, con el pico en el 2014. Una percepción de ellas, a través de los Títulos, se aprecia que mayoritariamente se refieren a Casos clínicos, y no necesariamente al esquema lógico de la Tesis de Grado.

Gráfico 1. Tesis de Bachiller y Doctoral. Registro numérico longitudinal retrospectivo F. Odontología UNMSM

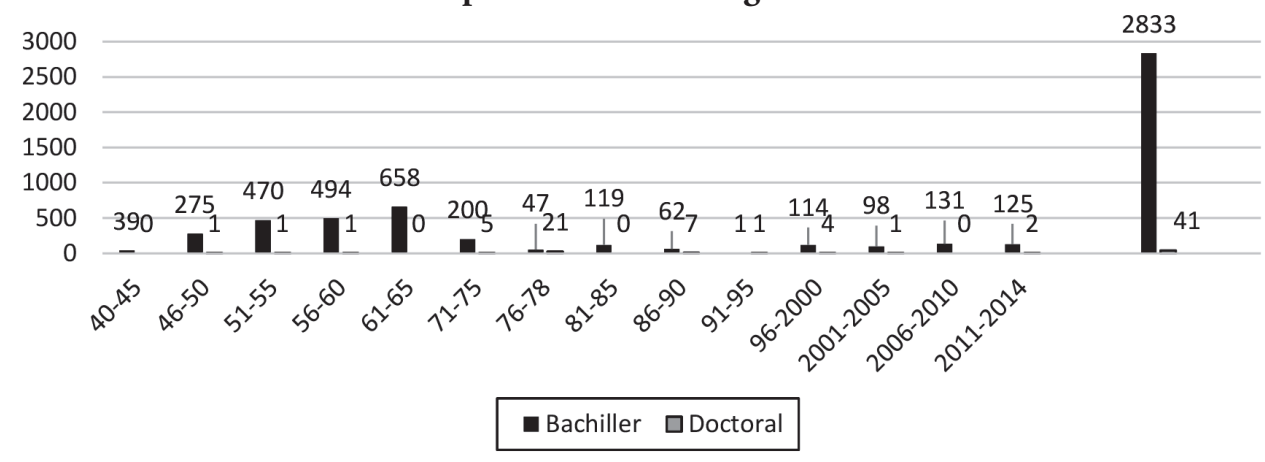

HIlda Morom Nakata

\section{Gráfico 2.Tesis de Maestría y Especialización. Registro numérico longitudinal retrospectivo.} F. Odontología UNMSM

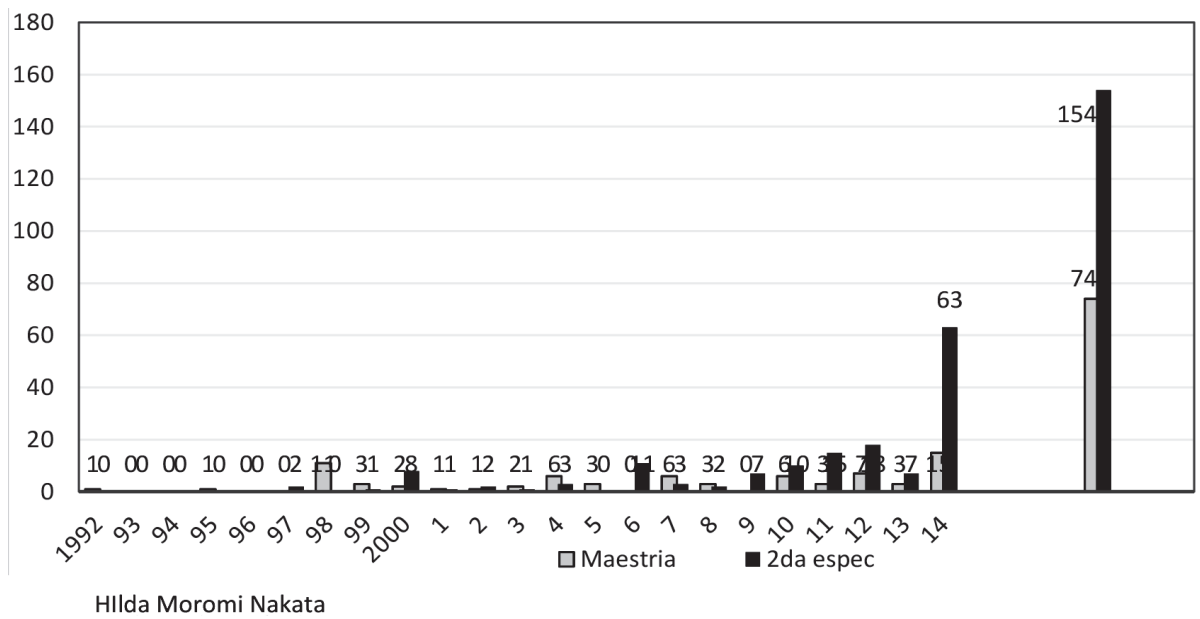

\section{Conclusión}

Este hallazgo simple y numérico es apenas la punta del iceberg de la enorme cantidad de conocimientos científicos contenidos en las miles de Tesis, almacenadas y poco aprovechadas en los anaqueles de una biblioteca. Iniciativas creativas y rigurosas pueden extraer multitud de conocimientos para sistematizar históricamente, y optimizar, la ciencia odontológica peruana, partiendo, por ejemplo, desde esquemas lógicos que manejen y operen válidamente la generación de los conocimientos, hasta la amplísima identificación de variables y su análisis y discusión, que encierran las miles de tesis señaladas; y que bien podrían ser materia para la maduración científica de los maestrandos y doctorandos. Por ejemplo, en 1940, la tesis de Elena Temoche abordaba la relación de la patología bucal y la gestación, seguramente en la inquietud de buscar desde ya la evidencia de una relación somato sistémica de la odontología: el hallazgo, sea cual fuere, es citado en las investigaciones similares. Debería, por ser la primera, y un referente del tema en la historia científica de la odontología peruana.

Por otro lado, de la revisión de los cuadros estadísticos en el Boletín de la Universidad y página web de la Facultad de Odontología en los últimos años se observan algunas discrepancias en los datos, lo que sugiere la necesidad de mejorar los trámites para que los documentos sustentados para la graduaciones y titulaciones se registren oportunamente en la sede de la biblioteca, para permitir la adecuada difusión de la producción científica institucional. 


\section{Agradecimiento:}

Al Blog http:/mrojas.perulactea.com/, por la cogida y publicidad los hallazgos.

\section{Referencias bibliográficas}

1. Rojas CM, Moromi NH. Aporte holístico para facilitar la comprensión de la Certificación universitaria. [Citado el 2015-12-10]. Disponible en: http://mrojas. perulactea.com/2010/09/03/ aporte-holistico-para-facilitarla-comprension-de-la-certificacion-universitaria/\#more-447 (20/12/2015).

2. Universidad Nacional Mayor de San Marcos. Facultad de Odon- tología. Hemeroteca. Catálogo de tesis 2014

3. Rojas CM. Tesis de Grado: Eficaz esquema lógico para el proyecto y la tesis. [Citado el 201512-20]. Disponible en http:// mrojas.perulactea.com/http:// mrojas.perulactea.com/wpcontent/uploads/2014/12/Tesis-oProyecto-Esquema-ModernoPDF.pdf

4. Rojas CM. Tipos de Investigación científica: Una simplificación de la complicada incoherente nomenclatura y clasificación. [Citado el 2015-12-20]. http:// mrojas.perulactea.com/http:// mrojas.perulactea.com/wpcontent/uploads/2015/01/Tipos-deInvestigaciu00F3n-2015-PDF.pdf

5. Rojas CM. Tipos de Investigación científica: Una simplificación de la complicada incoherente nomenclatura y clasificación. [Citado el 20/12/2015]. Disponible en: http:/vetcomunicaciones.com.ar/ uploadsarchivos/tipos_de_investigaciu00f3n_2015_pdf.pdf

6. Universidad Nacional Mayor de San Marcos. Facultad de Odontología. [Citado el 20/12/2015]. http://odontologiaunmsm.edu.pe/ pdf/2014/HISTORIAL_DE_TESIS_SUTENTADAS_2013.pdf 